\title{
Corpus
}

\section{Les mécanismes persuasifs des textes politiques}

Propositions théoriques pour l'analyse de corpus

\section{Carine Duteil-Mougel}

\section{CpenEdition}

\section{Journals}

Édition électronique

URL : http://journals.openedition.org/corpus/357

DOI : $10.4000 /$ corpus.357

ISSN : 1765-3126

Éditeur

Bases; corpus et langage - UMR 6039

Édition imprimée

Date de publication : 1 décembre 2005

ISSN : 1638-9808

\section{Référence électronique}

Carine Duteil-Mougel, « Les mécanismes persuasifs des textes politiques », Corpus [En ligne], 4 | 2005,

mis en ligne le 01 septembre 2006, consulté le 08 septembre 2020. URL : http://

journals.openedition.org/corpus/357 ; DOI : https://doi.org/10.4000/corpus.357

Ce document a été généré automatiquement le 8 septembre 2020.

(c) Tous droits réservés 


\title{
Les mécanismes persuasifs des textes politiques
}

\author{
Propositions théoriques pour l'analyse de corpus
}

\section{Carine Duteil-Mougel}

\section{Introduction}

1 La notion de corpus reçoit en linguistique différentes acceptions. Le corpus peut être défini comme une collection de données langagières ou comme un échantillon de langage; il peut également être conçu comme un ensemble de mots (ou "sac de mots" selon François Rastier, qui critique ce type de corpus issu de la tradition logicogrammaticale), ou comme un ensemble d'énoncés (ou «sac de phrases», toujours selon Rastier ${ }^{1}$ ), ou encore, dans notre perspective, comme un ensemble de textes.

2 Or tout ensemble de textes (critère de forme) n'est pas un corpus; des critères de fond entrent en jeu. Pour Bénédicte Bommier-Pincemin (1999: 416), le corpus doit vérifier trois types de conditions :

- Des conditions de signifiance : Un corpus est constitué en vue d'une étude déterminée (pertinence), portant sur un objet particulier, une réalité telle qu'elle est perçue sous un certain angle de vue (et non sur plusieurs thèmes ou facettes indépendants, simultanément) (cohérence).

- Des conditions d'acceptabilité : Le corpus doit apporter une représentation fidèle (représen-tativité), sans être parasité par des contraintes externes (régularité). Il doit avoir une ampleur et un niveau de détail adaptés au degré de finesse et à la richesse attendue en résultat de l'analyse (complétude).

- Des conditions d'exploitabilité: Les textes qui forment le corpus doivent être commensurables (homogénéité). Le corpus doit apporter suffisam-ment d'éléments pour pouvoir repérer des comportements significatifs (au sens statistique du terme) (volume).

3 Nous suivons François Rastier, qui propose la définition suivante du corpus (Rastier 2004a - c'est nous qui soulignons) :

Un corpus est un regroupement structuré de textes intégraux, documentés, éventuellement enrichis par des étiquetages, et rassemblés: (i) de manière théorique réflexive en tenant compte des discours et des genres, et (ii) de manière pratique en vue d'une gamme d'applications. 
4 Rastier adopte une conception praxéologique du texte selon laquelle tout texte relève d'un genre et tout genre, d'un discours, attaché à une pratique sociale. Selon Rastier, les recherches en sémantique des textes doivent porter sur des corpus aussi homogènes que possible pour ce qui concerne leur genre, ou du moins leur discours. Comme l'auteur le souligne, un texte peut "perdre » du sens, s'il est placé parmi des textes oiseux, car la comparaison avec eux ne permet pas de sélectionner d'oppositions pertinentes. D'autre part, le mélange des genres (corpus hétérogène) annule leurs spécificités et empêche leur caractérisation.

5 Aussi, disposer de critères pour la classification des textes, des genres et des discours apparaît comme un enjeu pour la linguistique de corpus et la constitution de corpus pleinement utilisables pour des tâches de description linguistique.

1. L'analyse des textes persuasifs

6 Notre travail participe au programme de caractérisation des textes, genres ${ }^{2}$ et discours ${ }^{3}$, et vise à proposer des critères pour leur typologie.

7 Notre attention se porte sur le fonctionnement persuasif des textes; et nous envisageons, dans le cadre d'une Sémantique-Rhétorique de corpus, les procédés sémantico-rhétoriques comme des outils de classification.

\subsection{Les composantes rhétoriques}

8 Nous proposons l'introduction de cinq composantes rhétoriques ${ }^{4}$ :

- la composante éthique

- la composante argumentative

- la composante pathétique

- la composante dispositionnelle

- la composante actionnelle ${ }^{5}$

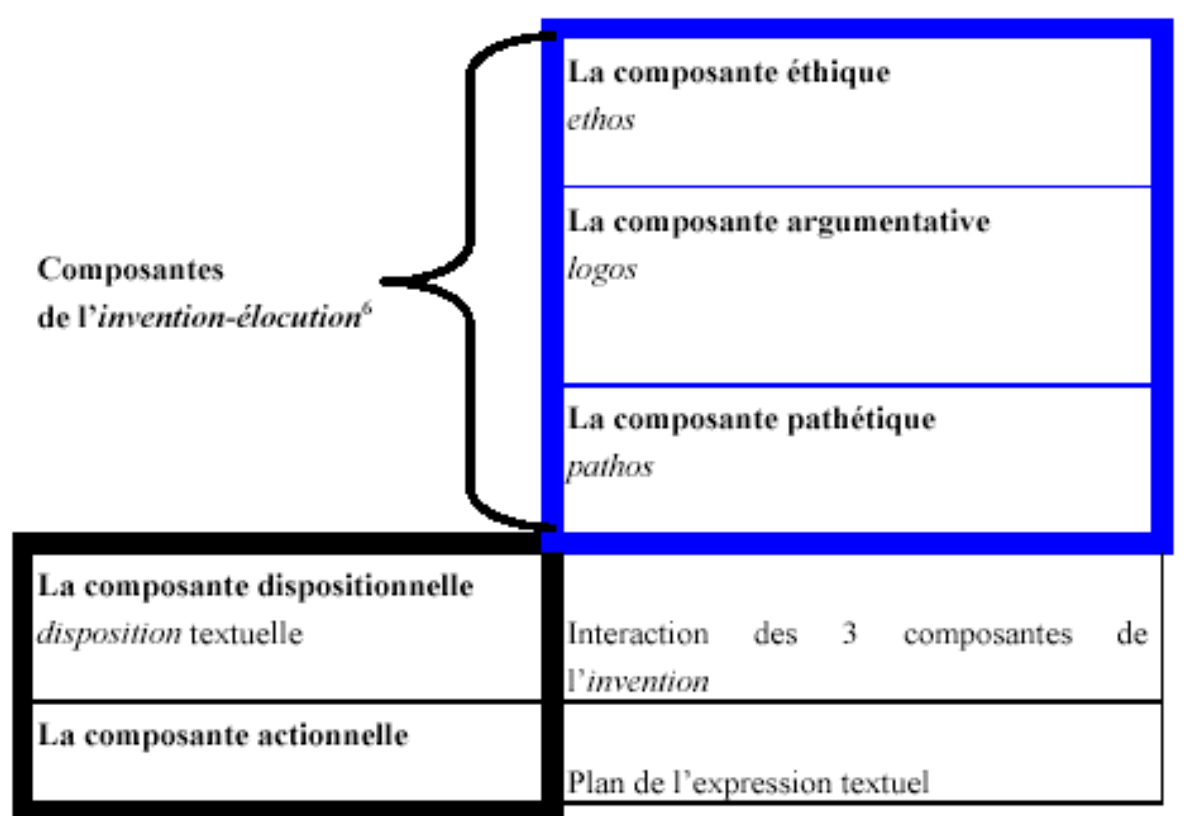

Relèvent ${ }^{6}$ de la composante éthique les procédés mis en œuvre par l'énonciateur pour se concilier la bienveillance de l'énonciataire, pour lui plaire, le charmer; ce sont par exemple des stratégies de séduction mais aussi des stratégies tonales ${ }^{7}$. 
9 Le recours aux figures non-tropes (par exemple, l'argument ad hominem, l'argument d'autorité, les questions rhétoriques, etc.), l'utilisation de procédés argumentatifs (par exemple, la gradation ascendante, les procédés d'insistance, etc.) mais aussi l'argumentation fondée sur des topoï (ou formes topiques) relèvent de la composante argumentative.

10 Relèvent de la composante pathétique des procédés visant à susciter l'émotion de l'énonciataire; il s'agit par exemple de l'utilisation de procédés d'amplification (comme la description hyperbolique, le recours à l'énumération, le style épithétique) mais aussi de procédés visant à sacraliser le discours de l'énonciateur.

11 La disposition textuelle est conçue comme l'ordonnancement de stratégies persuasives au sein du texte (tactique d'agencement textuel).

12 Participent de stratégies d'action les stratégies sur le plan de l'expression du texte (typodisposition ${ }^{9}$, distribution ${ }^{10}$, typographie, ponctuation, etc.).

1.2. Les normes génériques

13 Nous faisons l'hypothèse que le genre du texte norme le type de stratégie persuasive (éthique, argumentative, pathétique) ainsi que la disposition textuelle.

14 L'analyse de la Profession de foi de J.M. Le Pen ${ }^{11}$, nous a permis par exemple d'identifier une disposition textuelle remarquable en quatre parties ${ }^{12}$ :

-(i) un exorde : stratégies éthiques (éloge personnel, «mœurs réelles», doxa affichée);

- (ii) une narration-accusation : stratégies pathétiques et argumentatives (susciter la colère, la peur de l'énonciataire et l'inciter à la vengeance ; argument ad populum, subjection, enthymème);

-(iii) une confirmation-péroraison : stratégies associant argumentation (arguments-valeurs) et tentation (pathos) ;

- et (iv) une conclusion : stratégie éthique (élocution; style); ces quatre parties étant configurées respectivement, aux deux premières pages de la Profession de foi, à la troisième page de la Profession de foi, à la quatrième page de la Profession de foi, et à la fin de la quatrième page de la Profession de foi (deux dernières lignes).

L'analyse comparative d'autres Professions de foi devrait permettre, à partir de l'identification et de la description des stratégies persuasives et des stratégies sur la disposition textuelle, de proposer une première caractérisation des normes du genre «Profession de foi ». Il s'agira notamment de confirmer ou d'infirmer l'hypothèse d'une disposition textuelle en quatre parties - hypothèse formulée à partir de l'analyse de la Profession de foi de J.-M. Le Pen.

Ces normes devront être comparées à celles d'autres genres politiques, comme l'«allocution», le "communiqué officiel», la "déclaration présidentielle», le "discours parlementaire», le "discours de parti » etc., afin d'établir des champs génériques - un champ générique ${ }^{13}$ étant défini comme « un groupe de genres en coévolution qui contrastent, voire rivalisent dans un champ pratique " (Rastier 2001: 297).

17 Le plein accès à de grands corpus politiques comme PoliText ${ }^{14}$, et le recours à des outils de traitement logométrique performants comme le logiciel Hyperbase ${ }^{15}$, présentent un intérêt majeur pour cette entreprise.

2. Analyse de cas 
18 Nous débuterons l'analyse détaillée de la Profession de J.-M. Le Pen ${ }^{16}$ par le repérage de stratégies éthiques, puis nous nous intéresserons aux stratégies argumentatives et aux stratégies pathétiques mises en œuvre par l'énonciateur.

\subsection{Stratégies éthiques}

19 Chez Aristote, la preuve éthique consiste pour l'orateur à se montrer sous un jour favorable; il doit chercher à plaire à son auditoire et doit chercher à transférer la confiance que l'auditoire lui accorde, sur le propos qu'il défend. C'est l'existence d'une morale commune (doxa) qui permet à l'orateur d'incarner dans son discours les vertus qui inspirent la confiance publique.

« On persuade par le caractère, quand le discours est de nature à rendre l'orateur digne de foi, car les honnêtes gens nous inspirent confiance plus grande et plus prompte sur toutes les questions en général, et confiance entière sur celles qui ne comportent point de certitude, et laissent une place au doute. Mais il faut que cette confiance soit l'effet du discours, non d'une prévention sur le caractère de l'orateur. [...] c'est le caractère qui, peut-on dire, constitue presque la plus efficace des preuves. "

(Aristote, Rhétorique I, 1356a, [1932], pp. 76-77, Les Belles Lettres)

20 L'ethos discursif décrit par Aristote correspond aux « mœurs oratoires » de l'orateur, i.e. à l'image qu'il donne de lui à travers son discours, par la façon même dont il exerce son activité oratoire. L'ethos pré-discursif renvoie quant à lui, à la réputation de l'orateur, à ses actions passées, à ses «mœurs réelles"; il précède ainsi l'activité oratoire de l'orateur et n'est pas construit par elle. Un tel ethos est à l'œuvre dans un système fondé sur l'auctoritas, celui que connaissent les Romains et dont témoigne l'un des arguments que Cicéron utilisait dans ses procès : « acquittez-le puisque c'est moi qui le demande ».

B. Gibert (La Rhétorique ou les règles de l'éloquence), cité par M. Le Guern (1977 : 284), définit la distinction entre « mœurs oratoires » et « mœurs réelles » :

« Nous distinguons les mœurs oratoires d'avec les mœurs réelles. Cela est aisé. Car qu'on soit effectivement honnête homme, que l'on ait de la piété, de la religion, de la modestie, de la justice, de la facilité à vivre avec le monde, ou que, au contraire, on soit vicieux, [...], c'est là ce qu'on appelle mœurs réelles. Mais qu'un homme paraisse tel ou tel par le discours, cela s'appelle mours oratoires, soit qu'effectivement il soit tel qu'il le paraît, soit qu'il ne le soit pas. Car on peut se montrer tel, sans l'être ; et l'on peut ne point paraître tel, quoiqu'on le soit; parce que cela dépend de la manière dont on parle. »

2.1.1. L'ethos rhétorique détourné

L'énonciateur-candidat - désormais noté E-C - débute son discours ${ }^{17}$ par son propre éloge en mettant en valeur son expérience et ses mérites. Il se met en avant, parle de lui, évoque sa condition sociale, ses origines, ses actions passées et ce faisant détourne l'ethos rhétorique puisqu'il ne s'agit à proprement parler ni d'un ethos discursif - ethos aristotélicien - ni d'un ethos pré-discursif - ethos des Latins -, comme nous le montrerons ci-dessous.

O. Ducrot (1984 : 200-201) décrit ce détournement de l'ethos rhétorique (c'est nous qui soulignons) :

«Un des secrets de la persuasion telle qu'elle est analysée depuis Aristote est, pour l'orateur, de donner de lui-même une image favorable, image qui séduira l'auditeur et captera sa bienveillance. Cette image de l'orateur, désignée comme ethos ou "caractère", est encore appelée quelquefois - l'expression est bizarre mais significative - «mœurs oratoires ». Il faut entendre par là les mœurs que l'orateur s'attribue à lui-même par la façon dont il exerce son activité oratoire. Il ne s'agit 
pas des affirmations flatteuses qu'il peut faire sur sa propre personne dans le contenu de son discours, affirmations qui risquent au contraire de heurter l'auditeur, mais de l'apparence que lui confèrent le débit, l'intonation, chaleureuse ou sévère, le choix des mots, des arguments (le fait de choisir ou de négliger tel argument peut apparaître comme symptomatique de telle qualité ou de tel défaut moral). Dans ma terminologie, je dirai que l'ethos est attaché à $\mathrm{L}$, le locuteur en tant que tel: c'est en tant qu'il est source de l'énonciation qu'il se voit affublé de certains caractères qui, par contrecoup, rendent cette énonciation acceptable ou rebutante. Ce que l'orateur pourrait dire de lui, en tant qu'objet de l'énonciation, concerne en revanche $\lambda$, l'être du monde, et ce n'est pas celui-ci qui est en jeu dans la partie de la rhétorique dont je parle [...]»

Ici l'E-C détaille ses « mœurs réelles » en les énonçant dans son discours; il se met luimême en avant et recourt à l'éthopée morale ou peinture morale, pour évoquer sa condition sociale, ses origines, ses convictions et ses actions passées. Son discours s'apparente davantage au témoignage-confidence, et on remarquera sur le plan de l'expression, l'écriture inclinée vers la gauche (de 10 degrés environ) et la signature manuscrite mise en évidence par la couleur de police bleu roi ${ }^{18}$.

L'image que l'E-C donne de lui se veut très positive; il cherche ainsi à influer sur l'opinion de l'énonciataire-électeur - désormais noté E-E - et à gagner sa confiance. Il construit sa compétence et montre qu'il est qualifié pour faire. Ici le faire correspond à présider la France dans le but de servir sa patrie et avec l'ambition de faire le bonheur des Français.

Puis l'E-C se présente comme un ancien combattant toujours aussi /déterminé/ ${ }^{19}$, / combatif/ et /patriote/ que par le passé.

Il se définit également comme un /homme du peuple/ - un /travailleur/, un /père de famille/ - et un /homme d'action/ (responsabilités sociales, responsabilités politiques).

On soulignera que l'E-C affiche d'emblée, dès la première page, sa détermination et sa volonté de se consacrer aux Français: «La France et les Français d'abord!». Cette accroche-slogan est mise en évidence sur le plan de l'expression par la taille de la police et la couleur de police jaune. L'E-C semble s'engager personnellement; cet effet est renforcé par l'utilisation d'une police de caractère proche de l'écriture manuscrite et par l'italique et l'orientation de l'écriture (de 5 degrés environ vers la gauche). L'association candidat-engagement est renforcée par la mise en valeur des nom et prénom du candidat due à l'utilisation de l'ombre portée, de l'italique, des traits épais, de la taille élevée de la police, et de la couleur de police jaune. L'utilisation des majuscules - l'équivalent de la voix forte à l'oral - pour le nom du candidat, associée à l'utilisation du point d'exclamation - ponctuation forte - dans l'accroche-slogan, crée un effet d'insistance.

9 Le visuel (photographie) quant à lui, sert d'interprétant pour l'actualisation des sèmes de l'acteur ${ }^{20}$ 'Jean-Marie Le Pen'. L'acteur représenté semble en mouvement (déplacement, élan) vers la droite (orientation vers la droite, épaule gauche coupée par le cadrage). La position de l'actant observateur ${ }^{21}$ (position assumée par l'énonciataire) est située en bas à gauche - on ne voit que l'épaule droite de l'acteur représenté. Le regard de l'acteur représenté est légèrement orienté vers la gauche et vers le bas en direction de l'actant observateur ; sa tête est légèrement inclinée vers la gauche. L'acteur représenté semble s'adresser à l'actant observateur.

Ces éléments permettent selon nous l'actualisation des sèmes afférents ${ }^{22}$ /déterminé/, / engagé/, /combatif/ sur l'acteur ‘Jean-Marie Le Pen'. 
31 La prise de vue effectuée de bas en haut, en contre-plongée, accorde la priorité à l'acteur représenté, au détriment de l'actant observateur; les sèmes afférents / imposant/, /puissant/ sont selon nous, actualisés sur l'acteur 'Jean-Marie Le Pen'.

2.1.2. La doxa affichée

Un topos doxique que l'on choisira de nommer Nationalisme - doxa censée partagée par l'E-E - est convoqué dès le slogan-accroche de la page 1. Ce topos est évalué positivement (fonds évaluatif) de façon explicite aux deux premières lignes de la page 2: «L'indépendance de la Patrie et la liberté des citoyens sont les biens les plus précieux du Peuple. » En énonçant cette maxime, l'E-C revêt l'ethos du moraliste (ethos discursif); il affiche ses préférences: la Patrie, la Nation, l'indépendance et la souveraineté de la France, l'identité du Peuple Français, etc.

Son positionnement est souligné à la fin de la page 2 par l'épiphrase (procédé d'insistance): "Vive la France!" mise en valeur sur le plan de l'expression par l'utilisation des traits épais et de la couleur de police bleu roi ${ }^{23}$.

2.2. Stratégie sur la disposition textuelle : l'exorde

Toutes les stratégies éthiques analysées précédemment apparaissent au début du discours de l'E-C. Cette première partie du discours s'apparente ainsi selon nous à l' exorde rhétorique ${ }^{24}$. L'exorde est une des six parties du discours définies dans les traités rhétoriques (disposition rhétorique); il correspond au début d'un discours et a pour fonction de disposer et de préparer l'esprit de l'auditoire-énonciataire à écouter l'orateur-énonciateur. C'est dans l'exorde que l'orateur-énonciateur cherche à attirer l'attention de l'auditoire-énonciataire sur ses mérites, et qu'il cherche à lui plaire et à se concilier sa bienveillance.

Nous repérons donc ici une stratégie sur la disposition textuelle visant à débuter le discours par des stratégies éthiques - procédés sémantico-rhétoriques repérés au sein de parcours interprétatifs. Cette stratégie sur la disposition est renforcée par une stratégie sur la distribution textuelle (plan de l'expression), l'exorde étant configuré aux deux premières pages de la profession de foi.

2.3. Stratégies argumentatives («logiques ») et stratégies pathétiques 2.3.1. Une vision négative de la situation de la France et des Français

36 Ici nous verrons que l'E-C recourt au pathos: il cherche à susciter chez l'E-E un sentiment de colère, mais aussi de peur, et l'incite à la vengeance.

37 Aristote définit les passions comme étant des agents de variation du jugement des auditeurs :

«Les passions sont les causes qui font varier les hommes dans leurs jugements et ont pour consécutions la peine et le plaisir, comme la colère, la pitié, la crainte, et toutes les autres émotions de ce genre, ainsi que leurs contraires. »

(Aristote, Rhétorique II, 1378 a, [1960], p. 60, Les Belles Lettres)

\section{a) Le complot :}

L'E-C évoque d'abord ${ }^{25}$ un " complot » dirigé contre lui, privant l'E-E de la liberté de choisir librement le président de la République Française. Un topos narratif que nous choisissons de nommer Complot est convoqué ; l'E-C dénonce cette tentative de complot et recourt à la figure du procès d'intention ${ }^{26}$ (« Ils ont peur qu'en m'entendant [...]») pour désigner les coupables, à savoir le « Président-candidat », " ses amis » et " Jospin ». L'E$C$ précise les raisons de l'existence du complot à l'aide de la subjection : l'E-C pose une 
question et se répond lui-même dans un simulacre de dialogue avec l'E-E (connivence avec l'auditoire) : «"Pourquoi ?" Parce que je dis [...]».

L'E-C rapporte également les paroles qui sont censées être celles de l'E-E (dialogisme interlocutif): «Ils ont peur qu'en m'entendant, vous soyez de plus en plus nombreux à vous dire "LE PEN a raison". » On notera l'effet d'insistance créé par l'utilisation des majuscules et par l'utilisation conjointe de la couleur de police bleu roi et des caractères gras (plan de l'expression).

En présentant ces raisons, l'E-C présuppose que l'existence de ce complot est avérée et n'en fournit aucune preuve ${ }^{27}$. L'inscription dans la marge "Le complot a échoué » (mise en valeur sur le plan de l'expression par l'utilisation de la couleur de police bleu roi et par la taille de police élevée) présuppose qu'il y avait bel et bien tentative de complot.

4 Un premier raisonnement (stratégie argumentative) concerne justement ce complot: n'est exprimée que la prémisse mineure d'un enthymème ${ }^{28}$ qui pourrait avoir pour prémisse majeure: priver du droit d'élire librement le président de la République est condamnable et pour conclusion: donc il faut condamner le Président-candidat et ses amis chiraquiens. Cette conclusion, c'est à l'E-E de la suppléer et de l'assumer.

\section{b) Le déclin de la France.}

L'E-C présente ensuite ${ }^{29}$ dans le détail la situation catastrophique dans laquelle sont censés se trouver la France et les Français. L'E-C évoque également ${ }^{30}$ les menaces qui pèsent sur eux et qui vont jusqu'à toucher la famille et l'école.

Cette situation est présentée comme réelle ; elle est attestée du point de vue de l'E-C (univers d'assomption ${ }^{31}$ ). Cette situation est dénoncée (évaluation négative) car elle porte atteinte aux valeurs du topos doxique Nationalisme (faisant appel ici à la devise du régime de Vichy: « Travail, Famille, Patrie »).

Le recours à l'énumération permet d'insister sur cette situation jugée comme étant / dysphorique/ et /menaçante/ pour la France et les Français. L'utilisation de l' accumulation concourt à rendre cette situation insupportable pour l'E-E et à amplifier son sentiment de colère à l'égard des gouvernants, accusés ici ouvertement d'être à l'origine de cette situation. On soulignera que l'intertitre - « La corruption règne à la tête de la République.»-, signalé par la couleur de police bleu roi et l'espacement (interligne), met en évidence l'accusation du Président de la République.

45 Nous repérons sur le plan thématique, le thème $\mathrm{e}^{32}$ de la Décadence de la France construit à partir de la molécule sémique suivante :

- PROCESSUS : /dégradation/, /privation/, /intensité/, /duratif/, /amplification/

- RESULTAT : /manque/, /dysphorie/

- “détrimentaires” : ‘France', 'Français'

- Sémies ${ }^{33}$ et contenus de syntagmes indexés :

'bilan désastreux', 'reculer', 'a perdu', 'démantelée', 'chômage', 'insécurité', 'fiscalisme', 'peur', ‘conséquences dramatiques', 'impuissantes', ‘s'installe', 'régressent', ‘s'alourdissent', 'bafouée, 'incapable', 'racket', 'drogue', 'violence', 's'installent', 'désespèrent', 's'angoissent', 'désordre', 's'installe', ‘menace', ‘sans emploi', 'menacés', ‘depuis 20 ans', 'ne cesse de', 'tant à l'intérieur qu'à l'extérieur', 'progressivement', 'plus grave encore', 'folle politique', 'immigration massive', 'de plus de', '10 millions d'étrangers du Tiers-monde', 'de plus en plus', 'trop souvent', 'trop tard' 


\section{c) L'urgence de l'action.}

Le moyen pour l'E-E de se venger est mis en évidence par la mention dans la marge : « Il faut voter national pour redonner la parole au Peuple $»^{34}$. Cette mention est soulignée par l'utilisation de la couleur de police bleu roi et des caractères gras.

Un effet d'insistance est créé aux lignes 50 et 54-55 par la mise en valeur (couleur de police bleu roi et caractères gras) des propositions : «Il n'est que temps de réagir, avant qu'il ne soit trop tard" et "il faut redonner la parole au Peuple et le consulter directement par référendum ». La proposition «Cette élection sera décisive et l'avenir dépend de votre vote. » (ligne 31) mise en évidence sur le plan de l'expression, participe de cette dramatisation et confère à l'E-E un rôle capital.

Nous proposons le raisonnement suivant qui sous-tend l'argumentation de l'E-C dans les deuxième, troisième et quatrième paragraphes de cette troisième page - ce raisonnement n'est pas à proprement parler un enthymème mais plutôt un enthymémisme $e^{35}$; on peut parler également d'argument ad populum :

- Prémisse majeure : Pour changer la situation menaçante dans laquelle se trouvent la France et les Français, il faut redonner la parole au Peuple

- Prémisse mineure : or voter national c'est redonner la parole au Peuple

- Conclusion : donc voter national c'est changer la situation menaçante dans laquelle se trouvent la France et les Français ${ }^{36}$.

\subsubsection{Le redressement de la France}

L'E-C présente ensuite ${ }^{37}$ son « programme de redressement national » dont les grandes lignes, mises en évidence par la couleur de police bleu bleuet ${ }^{38}$ et les caractères gras (plan de l'expression), sont présentées dans les marges de la quatrième page et reprises $^{39}$ à chaque début de paragraphe ${ }^{40}$. L'E-C exploite dans son programme d'action, le topos doxique Nationalisme et la devise "Travail, Famille, Patrie ».

$50 \mathrm{Au}$ niveau des stratégies éthiques, on notera l'utilisation de termes techniques (juridiques, économiques) renforçant la crédibilité, le sérieux de l'E-C - par exemple : «abrogation», «droit d'asile», «35\% du produit national», « retraite par répartition », « régime optionnel de capitalisation ».

51 Sur le plan thématique, nous repérons le thème du Redressement de la France construit à partir du faisceau isotopique ${ }^{41}$ suivant :

1) - PROCESSUS : /retour à/

- RÉSULTAT : /euphorie/

- bénéficiaire : 'France'

Sémies et contenus de syntagmes indexés:

'restaurer', 'rendre', 'reprendre', 'restauration', 'rétablir', 'rétablissement'(x2),

'réinscrire', 'redevenir', 'relance', 'à nouveau', 'capital', 'hérité'

2) - PROCESSUS : /restriction/, /privation/, /intensité/, /dégradation/

- "maléficiaires” : 'Europe', 'Délinquants', 'Immigrés', 'Culture de la mort'

Sémies et contenus de syntagmes indexés :

'dénonciation', 'tolérance zéro', 'sanction pénale', 'peine de mort', 'prisons', 'maisons de correction', 'expulsion', 'abrogation', 'restriction', 'contrôles', 'exclusivement', 'préférence nationale'(x2), 'réduire', 'fortement', 'limitant', 'triompher sur'.

3) - PROCESSUS : /amélioration/

- RÉSULTAT : /euphorie/

- bénéficiaires: 'France', 'Français', 'Familles Françaises', 'Nature', 'Vie', 'Créatures vivantes' 
Sémies et contenus de syntagmes indexés :

'souveraineté', 'indépendance', 'unité', ‘Europe des nations', 'indépendance monétaire', 'ordre', 'loi', 'préférence nationale'(x2), 'emploi', 'grâce à', 'nos petites et moyennes entreprises', 'développement', 'prospérité', 'tenir leur place', 'source de création d'emplois', 'famille', 'priorité, natalité', 'enfant', 'avantages', 'familles françaises', 'revenu parental', 'sauver', 'retraites', 'développement', 'natalité, 'défendre', 'nature'(x2), 'vie', 'triompher', 'défendant', 'véritable écologie', 'protection', 'créatures vivantes', 'exceptionnel', 'capital de civilisation'. susciter le désir de l'E-E (stratégie pathétique). Ce désir peut être d'autant plus fort que la crainte des menaces évoquées par l'E-C juste avant, n'a pas disparu. L'E-E pourrait ainsi être amené à voter en faveur du candidat Front National afin de satisfaire son désir et dissiper ses craintes.

2.4. Stratégies sur la disposition textuelle : la narration-accusation et la confirmationpéroraison Après avoir débuté son discours par des stratégies éthiques, l'E-C met en œuvre des stratégies pathétiques et «logiques ». Son discours est alors structuré en deux parties consécutives, la première s'apparente selon nous à la narration-accusation rhétorique, une autre partie de la disposition qui consiste à exposer les faits en désignant les coupables. Il s'agit ainsi, de notre point de vue, d'une stratégie sur la disposition textuelle visant à poursuivre le discours par des stratégies pathétiques et «logiques » procédés sémantico-rhétoriques repérés au sein de parcours interprétatifs. associons ici : la confirmation et la péroraison. La confirmation présente les arguments de l'orateur-énonciateur; la péroraison a plusieurs fonctions: elle met l'auditeurénonciataire en de bonnes dispositions pour l'orateur-énonciateur, elle amplifie, elle excite les passions chez l'auditeur-énonciataire, enfin elle récapitule les arguments. Il s'agit ainsi de notre point de vue, d'une autre stratégie sur la disposition textuelle visant à terminer le discours par des stratégies mêlant argumentation et tentation (pathos) procédés sémantico-rhétoriques repérés au sein de parcours interprétatifs.

Ces stratégies sur la disposition sont toutes deux renforcées par des stratégies sur la distribution textuelle (plan de l'expression): la narration-accusation rhétorique est configurée à la troisième page de la profession de foi, alors que la confirmationpéroraison est configurée à la quatrième page de la profession de foi.

2.5. Tactique

Nous ferons une remarque sur le plan de la composante tactique (tactique dialogiquethématique) : le monde factuel /dysphorique/ - celui de la Décadence de la France - est présenté juste avant le monde possible /euphorique/ - celui du Renouveau, du Redressement de la France.

Les thèmes de la Décadence de la France et du Redressement de la France étant selon nous en relation d'antonymie ${ }^{42}$, nous considérons qu'une transformation thématique - ou " métamorphisme $»^{43}$ - s'opère ainsi entre la narration-accusation (configurée à la page 3) et la confirmation-péroraison (configurée à la page 4).

L'E-C termine ainsi son discours par l'exposé d'une vision positive de la France à laquelle est censé adhérer l'E-E. Cette vision laisse entrevoir à l'E-E ce que pourrait être la France de demain, et l'on notera sur le plan intertextuel, que le titre de la profession de foi du candidat J.-M. Le Pen pour le second tour de l'élection présidentielle ( $\mathrm{La}$ 
France retrouvée ») indique vraisemblablement que le changement est en route - et ce grâce aux suffrages apportés par les Français lors du premier tour (le « vote national »). 2.6. Stratégie d'élocution corrélée à une stratégie sur la disposition textuelle

L'E-C achève son discours (lignes 91 et 92) par une sentence prenant la forme d'un épiphonème. Ces deux dernières phrases sont d'un style recherché (élocution) : on repère une anaphore (" Tout ce que »), une épiphore («vous »), des reprises (polyptotes : « a été » - « sera »; « ai fait » - « ferai ») mais aussi un parallélisme structurel qui met en évidence le changement de préposition (" pour vous»; « avec vous»). Notons que l'utilisation de la préposition « avec » permet d'associer l'énonciataire au combat de l'énonciateur.

Grâce à l'effet de parallélisme, ces deux phrases peuvent être facilement mémorisées par l'E-E et peuvent marquer son esprit. On notera leur mise en valeur sur le plan de l'expression, par l'espacement (interligne) et par l'utilisation de la couleur de police bleu bleuet et des caractères gras.

61 Cette sentence finale s'apparente selon nous à la partie de la disposition rhétorique appelée conclusion - la conclusion clôt avec art le discours. Nous repérons donc ici une stratégie sur la disposition textuelle visant à clôturer le discours par une stratégie d' élocution. Cette stratégie sur la disposition est renforcée par une stratégie sur la distribution textuelle (plan de l'expression), la conclusion étant configurée à la fin de la dernière page de la profession de foi.

Conclusion

62 Nous avons centré notre analyse sur l'identification et la description de stratégies persuasives et de stratégies sur la disposition textuelle.

63 Comme nous le précisions au début de cet article, ce travail participe de recherches menées dans le cadre d'une Sémantique-Rhétorique de corpus, visant à établir des critères pour la typologie des textes, des genres et des discours persuasifs (typologie qui paraît indispensable pour les traitements automatiques).

Dans cette perspective, il s'agit pour nous d'élaborer dans un premier temps, un codage XML des unités sémantico-rhétoriques des textes persuasifs - jeu de balises, règles d'enchaînement des balises, choix d'encodage.

Nous faisons ci-après, en guise de conclusion, quelques propositions ${ }^{44}$ pour le balisage de corpus :

\begin{tabular}{|c|c|}
\hline Types de balises & Candidats balises - exemples \\
\hline Balises Stratégie & $\begin{array}{l}<\text { ethos }><\text { /ethos }> \\
<\operatorname{logos}></ \text { logos }> \\
<\text { pathos }></ \text { pathos }>\end{array}$ \\
\hline Balises Argumentation & $\begin{array}{l}\text { <gradation_ascendante }>\text { /gradation_ascendante> } \\
\text { <enthymeme></enthymeme> } \\
\text { <argument_a_contrario></argument_a_contrario> }\end{array}$ \\
\hline
\end{tabular}




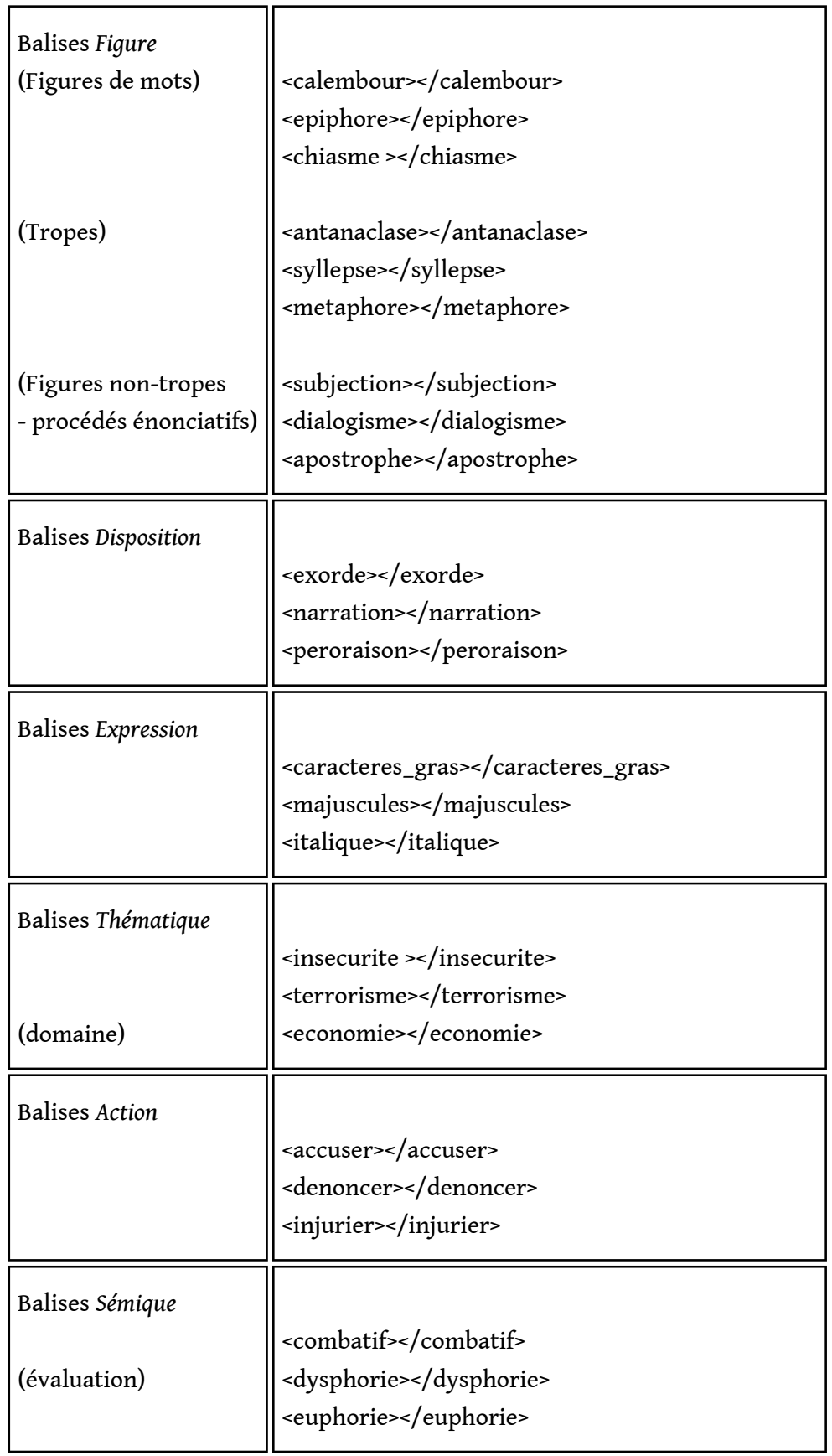

\section{BIBLIOGRAPHIE}

Amossy R. (dir.) (1999). Images de soi dans le discours. La construction de l'ethos. Lausanne / Paris : Delachaux \& Niestlé.

Amossy R. (2000). L'argumentation dans le discours. Paris : Nathan 
Aristote [1932]. Rhétorique I, 1356a. Paris : Les Belles Lettres.

Aristote [1960]. Rhétorique II, 1378 a. Paris : Les Belles Lettres.

Bakhtine M. (1984). Esthétique de la création verbale. Paris : Gallimard.

Bommier-Pincemin B. (1999). Diffusion ciblée automatique d'informations : conception et mise en cuvre d'une linguistique textuelle pour la caractérisation des destinataires et des documents. Thèse, Paris IV.

Bonnafous S., Chiron P., Ducard D. \& Levy C. (éds.) (2003). Argumentation et discours politique. Rennes : PU Rennes.

Burnard L. \& Sperberg-Mcqueen C. M. (éds) (2002). TEI P4 : guidelines for Electronic Text Encoding and Interchange (XML-compatible edition) 2 vol., University of Virginia Press.

Ducrot O. (1984). Le Dire et le dit. Paris : Minuit.

Dupriez B. (1984). Gradus, les procédés littéraires (diction-naire). Paris : Union générale d'éditions.

Duteil-Mougel C. (2004). Persuasion et Textualité. Propositions pour l'analyse sémantique et rhétorique de textes persuasifs. Thèse, Université Toulouse II, $614 \mathrm{p}$.

Fontanier P. [1977] (1821). Les figures du discours (introd. par G. Genette). Paris : Flammarion.

Greimas A. J. \& Courtés J. (1993). Sémiotique, dictionnaire raisonné de la théorie du langage. Paris : Hachette.

Habert B., Fabre C. \& Issac F. (1998). De l'écrit au numérique : constituer, normaliser, exploiter les corpus électroniques. Paris : InterÉditions / Masson.

Habert B., Nazarenko A. \& Salem A. (1997). Les linguistiques de corpus. Paris : Armand Colin / Masson.

Ide N. \& Véronis J. (1998). «Introduction to the Special Issue on Word Sense Disambiguation : the State of the Art », in N. Ide \& J. Véronis (éds.) Computational Linguistics 24, 1 : 1-40.

Le Guern M. (1977). «L'ethos dans la rhétorique française de l'âge classique », in C.R.L.S. (éd.) Stratégies discursives. Lyon : PU Lyon.

Malrieu D. \& Rastier F. (2001). « Genres et variations morphosyntaxiques », Traitement Automatique des langues 42, $2:$ 548-577.

Mayaffre D. (2000). Le poids des mots. Le discours de gauche et de droite dans l'entre-deux-guerres. Maurice Thorez, Léon Blum, Pierre-Etienne Flandin et André Tardieu (1928-1939). Paris : Honoré Champion.

Plantin C. (1990). Essais sur l'argumentation. Paris, Kimé.

Rastier F. (2001). Arts et sciences du texte. Paris : PUF.

Rastier F. (2004). " Doxa et lexique en corpus - pour une sémantique des "idéologies" ", in Actes des Journées Scientifiques en linguistique 2002-03, CIRLLLEP 22.

Rastier F. (2005). « Enjeux épistémologiques de la linguistique de corpus », in G. Williams (éd.) La linguistique de corpus. Rennes : PU Rennes, pp. 31-45.

Rastier F., Cavazza M. \& Abeillé A. (1994). Sémantique pour l'analyse. Paris : Masson.

Rastier F. (éd.) (1995). L'analyse thématique des données textuelles : l'exemple des sentiments. Paris : Didier érudition.

Souchard M. et al. (1997). Le Pen Les mots. Paris : Le Monde Editions. 


\section{NOTES}

1.. "L'objet empirique de la linguistique est fait de textes oraux ou écrits, non de mots ou de phrases - qui ne s'observent pas à l'état isolé, et, même quand on les isole, restent toujours relatifs à un genre et un discours. » (Rastier 2004a).

2.. Les critères de genre sont d'abord sémantiques. Rastier propose l'hypothèse suivante : sur le plan sémantique, les genres sont définis par des interactions normées entre les composantes sémantiques (thématique, dialectique, dialogique, tactique) - pour une présentation des composantes, cf. Rastier 2001 : 38-41.

3.. À chaque discours est associé un sociolecte (ensemble d'usages linguis-tiques codifiés) et des normes doxiques (doxa discursive).

4.. En référence aux parties de la rhétorique (invention, élocution, disposition, action) et aux preuves techniques de la rhétorique aristotélicienne (ethos, logos, pathos). Cf. l'auteur (2004) pour une présentation de la refondation de la Rhétorique au sein de la Sémantique interprétative.

5.. En référence à l'action oratoire.

6. Nous associons sur le schéma l'inventio et l'elocutio de la Rhétorique antique dans la mesure où selon nous, les procédés d'élocution s'inscrivent dans des stratégies d' invention.

7.. Ton adopté par l'énonciateur, qui lui permet de se positionner vis-à-vis de son auditoire.

8.. Les figures de pensée de la tradition Rhétorique.

9.. Espaces, alinéas, titres, intertitres, etc.

10.. Paragraphes, pages, chapitres.

11.. Pour le premier tour de l'élection présidentielle de 2002.

12.. Cf. infra l'analyse détaillée de ce texte (\$2. Analyse de cas).

13.. Par exemple, au sein du discours littéraire, le champ générique du théâtre se divisait en comédie et tragédie ; au sein du discours juridique, les genres oraux constituent un champ générique propre (réquisitoire, plaidoirie, sentence).

14.. Laboratoire « Bases, Corpus et Langage » - UMR 6039, CNRS-ILF-UNSA, Nice http:// www.unice.fr/ILF-CNRS/politext

15.. Hyperbase est un logiciel d'exploitation documentaire et de traitement quantitatif des grands corpus textuels, créé par Étienne Brunet (laboratoire « Bases, Corpus et Langage $»)$.

http://ancilla.unice.fr/ brunet/pub/hyperbase.html

16.. Le texte analysé figure en annexe.

17.. Distribution textuelle : page 2 de la profession de foi.

18.. Bleu roi ou bleu France : bleu moyen soutenu, correspondant au bleu du drapeau français.

19.. Les sèmes ou traits de sens sont notés entre barres obliques.

20.. Un acteur est défini par des traits sémantiques. Il relève de la dialectique (la dialectique est l'une des quatre composantes sémantiques définies par Rastier ; elle renvoie à la narrativité ; cf. notamment Rastier 2001 : 38-41). Notation 'acteur'.

21.. Nous faisons référence à la notion d'observateur définie par la Sémiotique de l'École de Paris : «On appellera observateur le sujet cognitif délégué par l'énonciateur et installé par lui, grâce aux procédures de débrayage, dans le discours-énoncé où il est chargé d'exercer le faire réceptif et, éventuellement, le faire interprétatif (...) » (Greimas A. J. \& Courtés J. 1993 : 259). 
22.. Un sème afférent est actualisé par instruction contextuelle.

23. L'utilisation de cette teinte de bleu renforce l'évocation de la souveraineté de la France.

24.. Et vraisemblablement à l'exorde du genre épidictique : le genre de l'éloge. Ici c'est l'orateur lui-même qui fait son propre éloge (détournement du genre épidictique).

25.. Distribution textuelle : page 3, premier paragraphe. On notera que cette page 3 comporte quatre paragraphes signalés par les traits de marge, les interlignes, et les inscriptions dans les marges.

26.. « Le procès d'intention est une accusation portant, non sur les faits, mais sur des intentions que l'on prête plus ou moins gratuitement à l'adversaire » (Dupriez 1984 :

23).

27.. « La preuve du complot, c'est justement qu'il est impossible de le prouver. » (Souchard et al. $1997: 74$ ).

28. De la forme : or le président-candidat et ses amis chiraquiens ont voulu priver du droit d'élire librement le président de la République.

29.. Distribution textuelle : page 3 , deuxième paragraphe.

30.. Distribution textuelle : page 3 , troisième paragraphe.

31.. Univers d'assomption : partie d'un univers sémantique composée des propositions attribuées à un acteur de l'énoncé ou de l'énonciation représentée (Rastier 2001 : 303).

32. Un thème peut être décrit comme une molécule sémique. Nous reprenons à Rastier (2001 : 300) la définition de la molécule sémique comme " groupement stable de sèmes, non nécessairement lexicalisé, ou dont la lexicalisation peut varier. ».

33.. Sémie : signifié d'une lexie.

34.. Mention qui fait semble-t-il appel au topos doxique du National-Populisme.

35.. « L'Enthymémisme consiste dans un rapprochement vif et rapide de deux propositions ou de deux termes, d'où résulte dans l'esprit une conséquence vive et frappante qui le saisit et l'entraîne d'une manière victorieuse. » (Fontanier [1977] (1821), pp. 382-383).

36.. Mais rien n'indique comment cette situation de la France et des Français peut changer.

37. Distribution textuelle : page 4.

38. Bleu bleuet ou bleu barbeau : bleu moyen légèrement fade (couleur de la fleur de bleuet).

39.. On remarquera l'ajout de l'adverbe « fortement » au début du quatrième paragraphe.

40.. Cette quatrième page compte huit paragraphes signalés par une puce et un interligne.

41.. Faisceau : ensemble d'isotopies induites par la récurrence des éléments d'une même molécule sémique.

42.. Antonymie de thèmes envisagée par Rastier (2001: 194).

43.. Rastier $2001: 46$.

44.. Ces propositions sont pour le moment à l'état embryonnaire. 


\section{RÉSUMÉS}

Notre propos s'inscrit dans le cadre d'une Sémantique-Rhétorique de corpus. Nous privilégions l'analyse de contenu sur corpus textuel et nous nous engageons sur la voie de la caractérisation des textes, genres et discours. Notre perspective est détaillée à travers l'analyse d'un texte politique. Nos propositions portent sur le repérage et la description de procédés sémanticorhétoriques, ainsi que sur leur codage au sein des textes (balisage du corpus).

The persuasive devices in the political texts. Theoterical proposals for corpora analysis.

Our research is in line with a rhetorical semantics of corpora. We favour content analysis on a textual corpus and we embark on a characterisation of texts, genres and discourses. Our perspective is detailed through the analysis of a political text. Our proposals bear on the location and the description of semantico-rhetorical devices as well as on their coding within texts (corpus tagging).

INDEX

Mots-clés : argumentation, corpus, ethos, genre, pathos., politique, procédés, rhétorique, sémantique, texte, typologie

\section{AUTEUR}

CARINE DUTEIL-MOUGEL

ATIlF 